\title{
T-mind: um Aplicativo Gamificado para Estímulo ao Desenvolvimento de Habilidades do Pensamento Computacional
}

\author{
Francisco Ittalo Ribeiro Pessoa ${ }^{1}$, Ana Liz Souto Oliveira de Araújo ${ }^{12}$, Wilkerson L. \\ Andrade $^{2}$, Dalton D. Serey Guerrero ${ }^{2}$ \\ ${ }^{1}$ Departamento de Ciências Exatas - Universidade Federal da Paraíba \\ Rua da Mangueira, s/n, Companhia de Tecidos \\ Rio Tinto 58.297-000 - Rio Tinto - PB - Brasil \\ ${ }^{2}$ Laboratório de Práticas de Software - Universidade Federal de Campina Grande \\ Av. Aprígio Veloso, s/n, SPLab, Bodocongó \\ 58429-900 - Campina Grande - PB - Brasil \\ \{francisco.itallo, analiz\} edce.ufpb.br, \\ \{wilkerson, dalton\} @ computacao.ufcg.edu.br
}

\begin{abstract}
Computational Thinking is a problem-solving approach exploring cognitive abilities in Computer Science. This work aims at promoting Computational Thinking through an App without requiring previous programming knowledge from the subjects. The result has pointed out that the current version can be suitable to stimulate data analysis, data collection, decomposition, abstraction, and data representation abilities. The usability evaluation has indicated that the gamification elements have produced engagement, and the interface had a positive assessment.
\end{abstract}

Resumo. Pensamento Computacional é uma abordagem de resolução de problemas que explora habilidades cognitivas da área de Ciência da Computação. $O$ objetivo deste trabalho é promover o Pensamento Computacional por meio de um aplicativo gamificado sem a obrigatoriedade de ensino ou conhecimento prévio em programação por parte dos usuários. Os resultados apontaram que a versão atual do aplicativo pode ser adequada para estimular as habilidades de análise de dados, coleta de dados, decomposição, abstração e representação de dados. A avaliação de usabilidade apontou que os elementos gamificados motivaram o engajamento dos alunos, bem como a interface foi avaliada de forma positiva.

\section{Introdução}

Pensamento Computacional (PC) pode ser compreendido como uma estratégia para resolução de problemas através de raciocínio lógico e formal estendendo faculdades cognitivas [Wing 2006]. De acordo com a Computer Science Teachers Association (CSTA) (2011), o PC envolve um conjunto de habilidades cognitivas, auxiliando as pessoas na resolução de problemas gerais do cotidiano. Conforme enfatizam França et al. (2014), várias iniciativas precursoras vêm sendo utilizadas com o intuito de estimular as habilidades do PC na educação básica. Exemplos dessas iniciativas são a robótica 
educacional, o ensino de programação, as oficinas de computação desplugadas e o uso de ambientes gamificados (jogos e aplicativos). Esse último está em constante evolução devido às vantagens de se usar ambientes gamificados nas mais variadas áreas de conhecimento.

A gamificação tem por significado o uso de mecânicas, estética ou pensamentos de jogos com o intuito de promover a participação de pessoas, motivar a ação e contribuir para a aprendizagem e resolução de problemas em diferentes contextos, como a educação [Kapp 2012]. A literatura nacional apresenta trabalhos que relatam o uso de ferramentas gamificadas para estímulo e/ou avaliação de habilidades do PC [Araújo et al. 2016], com destaque para os jogos digitais que promovem o ensino de conceitos da computação e ensinos de algoritmos ou ambientes voltados para o ensino de programação. Ressaltamos que esses trabalhos se baseiam na abordagem de ensinar programação introdutória para estimular e/ou avaliar habilidades do PC. Nesse caso, é necessário que muitas vezes o estudante aprenda programação e expresse habilidades do PC por meio da atividade de codificar.

Apesar de se encontrar na literatura nacional trabalhos que relatam o uso de ambientes gamificados para o estímulo do PC, quando trazemos essa abordagem para o contexto de dispositivos móveis (smartphones e tablets) percebemos que ainda é tímido o número de trabalhos para essa área em específico, levando em consideração o crescimento no uso de dispositivos móveis. Em pesquisa divulgada recentemente pelo Instituto Brasileiro de Geografia e Estatística (IBGE), o celular atualmente torna-se a principal porta de acesso para a Web no Brasil, superando o computador ${ }^{1}$. O destaque maior são os dispositivos com o sistema operacional Android, que de acordo com a International Data Corporation (IDC) (2017), 95,5\% dos smartphones vendidos no Brasil são Android. Seguindo essa tendência, estimular habilidades relacionadas ao PC por dispositivos móveis parece ser uma alternativa promissora.

Diante desse contexto, o objetivo deste trabalho é viabilizar o estímulo do Pensamento Computacional por meio de um aplicativo gamificado chamado de T-mind sem a obrigatoriedade de ensino ou conhecimento prévio em programação por parte dos usuários. O T-mind estimula o estudante a resolver problemas que exploram habilidades do PC sem precisar de conhecimentos em programação, utilizando elementos gamificados os quais ajudam no engajamento da resolução das questões. Com esse objetivo em vista, pretende-se responder à questão de pesquisa geral deste trabalho: $O$ aplicativo gamificado proposto pode ser adequado para estímulo de habilidades do PC? Assim, este trabalho apresenta o processo de concepção, implementação, aplicação e avaliação do aplicativo.

O T-mind foi construído para o sistema operacional Android inspirado no aplicativo Duolingo, famoso aplicativo usado para o ensino de idiomas. A metodologia empregada neste trabalho mostra a maneira como o aplicativo foi construído, apresentando como as habilidades do $\mathrm{PC}$ foram inseridas e validadas no contexto do $\mathrm{T}$ mind e a avaliação de usabilidade do mesmo, realizada em uma amostra de alunos do Ensino Médio de uma escola pública, com o intuito de medir a motivação do usuário para

\footnotetext{
${ }^{1}$ http://www.ibge.gov.br/home/estatistica/populacao/acessoainternet2014/default.shtm
} 
VI Congresso Brasileiro de Informática na Educação (CBIE 2017)

Anais do XXVIII Simpósio Brasileiro de Informática na Educação (SBIE 2017)

resolver as questões do aplicativo.

Assim, as demais seções do artigo estão organizadas da seguinte forma: a Seção 2 apresenta algumas habilidades do PC identificadas na literatura; a Seção 3 discute alguns trabalhos relacionados; a Seção 4 descreve os procedimentos e métodos para atingir o objetivo do trabalho; a Seção 5 apresenta o aplicativo T- mind; a Seção 6 reporta e discute os resultados; por último, a Seção 7 apresenta as considerações finais como também os trabalhos futuros.

\section{Habilidades relacionadas ao PC}

O Pensamento Computacional consiste em um conjunto de habilidades e técnicas para resolução de problemas utilizadas por profissionais da computação no desenvolvimento de sistemas complexos [Wing 2006]. De acordo com Barr e Stephenson (2011), a lista de habilidades associadas ao PC é extensa e pode variar conforme a literatura.

A CSTA e a International Society for Technology in Education (ISTE) listam um conjunto de nove habilidades aplicadas no contexto da educação norte-americana que são conhecidas como núcleo do PC [Bar \& Stephenson 2011]: Abstração: capacidade de filtrar informações essenciais e descartar as informações desnecessárias em um determinado contexto; Decomposição: quebrar um problema grande em subproblemas, facilitando sua solução; Automação: utilizar a tecnologia como meio na resolução de tarefas repetitivas; Coleta de Dados: encontrar os dados necessários para resolver um problema; Representação de Dados: usar estruturas de dados, como tabelas e gráficos, para apresentar os dados; Análise de Dados: atribuir sentido a dados, encontrando padrões e conclusões; Construção de Algoritmo: sequências de instruções para alcançar um objetivo; Paralelização: identificar atividades que podem ser executadas em paralelo; Simulação: Usar ou criar simulações para experimentos.

\section{Trabalhos relacionados}

O trabalho de Pinho et al. (2016) relata o projeto, a implementação e a aplicação de um jogo para dispositivos móveis com intuito de estimular a habilidade de coleta de dados. A metodologia empregada visa estimular a habilidade através dos elementos gamificados presentes no jogo, que foi concebido por meio de uma atividade desplugada chamada de Caça ao Tesouro. A avaliação foi realizada por três turmas de estudantes do ensino fundamental em duas etapas: a primeira, avaliando a atividade aplicada de forma tradicional e a segunda, por meio de um teste de usabilidade da ferramenta. Como resultados, os autores constataram que a atividade Caça ao Tesouro aplicada com aparatos tecnológicos obteve resultados superiores em relação a aplicação tradicional.

O trabalho de Gomes e Alencar (2015) analisa, de maneira empírica, três jogos educacionais para dispositivos móveis voltados à promoção do PC. Os autores apontaram que, para disseminação do PC na educação básica, os jogos teriam que possuir algumas das seguintes indicações: sequenciamento, reconhecimento de padrões, loops, condicionais, funções e parâmetros. Os resultados apontaram que os três jogos possuem esses critérios, os quais são considerados relevantes para o desenvolvimento do PC.

Já Falcão e Barbosa (2015) apresentaram um estudo de caso do jogo mobile Light Boot 2, utilizando avaliação formativa e objetiva, bem como analisando os aspectos 
técnicos e pedagógicos da ferramenta. A avaliação formativa foi feita por crianças, analisando as possibilidades de aprendizagem por descoberta através de interação exploratória. Já a objetiva foi realizada utilizando heurísticas de usabilidade de softwares, como uma forma complementar à avaliação formativa. Os resultados apresentaram que os aspectos de interação exploratória, de metáforas visuais da interface e de design de interação, podem se tornar parâmetros para a avaliação de jogos para o desenvolvimento do PC.

Nosso trabalho se difere dos demais pois desenvolvemos e avaliamos um aplicativo gamificado para estimular habilidades do PC sem a obrigatoriedade de conhecimentos em programação por parte dos usuários. Os demais trabalhos encontrados buscam desenvolver o PC por meio de jogos digitais, através de noções de programação e pensamento algorítmico.

\section{Procedimento e Métodos}

Para atingir o objetivo do trabalho, como também responder à questão de pesquisa, realizamos 4 etapas. A primeira delas foi a concepção do T-mind (Seção 4.1) que englobou o processo de levantamento de requisitos e características, definição de interface e desenvolvimento da ferramenta. A segunda etapa foi a elaboração das questões (Seção 4.2), envolvendo a criação de questões do T-mind. A terceira etapa foi a validação das questões (Seção 4.3), a qual analisou e classificou quais habilidades do PC estão presentes no T-mind e por último, a etapa de avaliação do T-mind (Seção 4.4), que avaliou a usabilidade da ferramenta.

\subsection{Concepção do T-mind}

A concepção do T-mind teve início com uma revisão bibliográfica no intuito de levantar as habilidades relacionadas ao PC e o estado da arte sobre softwares, jogos e/ou aplicativos para estimular essas habilidades. Até a presente data não foram encontrados trabalhos que relatam a existência de aplicativos gamificados para estimular o PC, que se aproxime ao projeto do T-mind. Se faz presente na literatura aplicativos que apoiam o ensino de programação como o CodSchool (https://www.codeschool.com/), mas sua proposta é diferente, pois o mesmo oferece cursos para ensinar linguagens de programação. As demais ferramentas encontradas são voltadas ao contexto da programação, como o Scratch (https://scratch.mit.edu/) e o App Inventor (http://ai2.appinventor.mit.edu/), ou aos jogos digitais, como o Ligthbot 2 (https://lightbot.com/flash.html).

Também foi pesquisado na literatura possíveis aplicativos educacionais gamificados com o intuito de ser inspiração para o T-mind. Levamos em consideração além da aceitação na literatura, a interface amigável e os elementos de gamificação presentes no aplicativo. Dentre os aplicativos encontrados, o que mais pareceu adequado a ser seguido foi Duolingo (https://pt.duolingo.com), pois ele tem elementos de gamificação, contendo mais de 10 milhões de usuários pelo mundo, sendo fácil de usar, e de acordo com os seus usuários, divertido [Leffa 2014].

Logo após a revisão bibliográfica, se deu início a elaboração do T-mind. Foram definidos os requisitos funcionais e não-funcionais do aplicativo diante dos elementos gamificados desejados. Depois, deu-se as etapas de prototipação, implementação, testes 
VI Congresso Brasileiro de Informática na Educação (CBIE 2017)

Anais do XXVIII Simpósio Brasileiro de Informática na Educação (SBIE 2017)

até a versão funcional do aplicativo.

\subsection{Elaboração das Questões}

$\mathrm{Na}$ atual fase da pesquisa, as questões do T-mind foram criadas de forma colaborativa. Optamos por esse modelo com a intenção de capturar diferentes percepções em relação às habilidades do $\mathrm{PC}$ de graduandos em cursos de Computação. Então, selecionamos 8 alunos concluintes, com bom desempenho acadêmico e com participação em projetos de pesquisa e de monitoria nos cursos de Licenciatura em Ciência da Computação e Bacharelado em Sistemas de Informação, ambos do Campus IV da Universidade Federal da Paraíba.

O processo de elaboração de questões foi divido em dois momentos. No primeiro, foi ofertado aos alunos um treinamento presencial com duração de 120 minutos sobre PC. O objetivo desse treinamento foi: $(i)$ explicar o conceito do PC, as variadas formas de se abordá-lo e as 9 habilidades associadas ao PC em conformidade com o CSTA; (ii) o escopo de questões do T-mind, explicando que elas poderiam ser objetivas, abertas ou de montar; (iii) definir, como padrão, que o estilo de questões do T-mind envolveria exercícios de resolução de problemas curtos ou médios, sem a necessidade de deixar exposto conteúdo da Computação ou dependências de linguagens de programação.

Logo após o treinamento, no segundo momento, foi disponibilizado um formulário online para que cada um dos 8 graduandos submetesse suas questões elaboradas e foi estabelecido o prazo para essa atividade. Também foi disponibilizado um material com uma seleção de questões da OBI (Olimpíada Brasileira de Informática) que exploram habilidades do PC para que os alunos tivessem alguma referência de modelos de questões. Ao final das submissões de questões, foi feita uma triagem. Foram desconsideradas questões que apresentaram explicitamente execução de cálculo complexo ou conteúdo de matemática e de programação. Ao final da triagem, as questões restantes foram analisadas segundo a qualidade do problema no contexto de estimular habilidades do PC e, após essa etapa, 20 questões foram selecionadas.

\subsection{Validação das Questões}

Com o objetivo de validar as questões elaboradas de forma colaborativa, adotamos neste trabalho uma abordagem de avaliação qualitativa baseada em análise de conteúdo das questões, realizadas por pesquisadores da área de PC (juízes). O objetivo é explorar a percepção de pesquisadores da área de $\mathrm{PC}$ no intuito de analisar o potencial das questões do aplicativo em relação ao estímulo das habilidades associadas ao PC.

Para a etapa de avaliação foi compartilhado um documento contendo as 20 questões selecionadas e um questionário com 4 pesquisadores do programa de pósgraduação da Universidade Federal de Campina Grande, todos da área de Pensamento Computacional. O questionário enviado estava organizado da seguinte forma: foi disponibilizado um enunciado explicando como o pesquisador deveria avaliar as questões e também uma lista de habilidades do PC e suas definições. Para cada questão do documento, o pesquisador deveria indicar quais habilidades associadas ao PC podem ser estimuladas naquela questão. Também foi disponibilizado um campo de texto para que ele sugerisse considerações em relação às questões, como por exemplo, mudança/correção no enunciado ou nas alternativas. Para que uma habilidade do PC fosse 
considerada válida na questão, a mesma deveria ser identificada por pelo menos 3 pesquisadores (votação majoritária).

\subsection{Avaliação do T-mind}

A última etapa consistiu na avaliação do T-mind, com o objetivo de medir a sua experiência de uso. Para essa avaliação, foi realizada uma adaptação da técnica de Software Usability Measurement Inventory (SUMI), a qual permite coletar dados sobre eficiência, apreensibilidade, controle, utilidade e satisfação, todas percepções subjetivas dos usuários frente as interações realizadas na ferramenta [Silva et al. 2016]. Dentre os itens abordados por SUMI, adotamos na nossa avaliação os de eficiência, controle e satisfação. Relacionamos à eficiência, o funcionamento geral do T-mind nos dispositivos; ao controle, controlar o acesso do T-mind; e à satisfação, a interface do T-mind. Além dos três itens abordados por SUMI, criamos uma quarta categoria de motivação ao usar o aplicativo, com o intuito de medir o engajamento dos elementos gamificados presentes no T-mind.

A avaliação foi realizada por 25 alunos do $1^{\circ}$ e $2^{\circ}$ anos do Ensino Médio de uma escola pública, com uma faixa etária entre 15 e 17 anos, sendo $53 \%$ do sexo masculino e $47 \%$ do sexo feminino. A amostra foi escolhida por conveniência. O processo de avaliação foi organizado em dois encontros com diferença de uma semana entre eles e contou com a ajuda de dois professores da escola que reuniram os alunos e monitoraram todo processo de avaliação. Houve a preocupação de testar o T-mind antes em diferentes dispositivos e foi constatado que o mesmo funciona em diferentes versões do Android, tendo apenas limitações de recursos gráficos nos dispositivos com versões inferiores a 5.0 do sistema operacional.

No primeiro encontro, os alunos foram reunidos no laboratório da escola com o objetivo de instalar o T-mind, explicar o seu funcionamento e apresentar como seria a avaliação. Foi explicado que eles teriam uma semana para explorar o aplicativo e, consequentemente, tentar resolver as questões. Os alunos que não possuíam dispositivos Android pessoais tiveram tablets emprestados pela escola. No segundo encontro, foi aplicado um questionário composto por 15 questões agrupadas nas 4 categorias selecionadas (eficiência, satisfação, controle e motivação), utilizando lápis e papel, com o objetivo de avaliar o T-mind. A Figura 1 apresenta duas questões desse questionário.

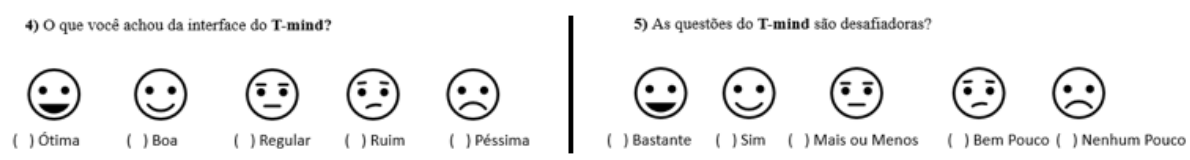

Figura 1. Duas questões do questionário aplicado

\section{O T-mind}

O T-mind é um aplicativo com elementos de gamificação que tem como foco estimular habilidades relacionadas ao PC, sem necessidade de conhecimento em programação. Para que isso aconteça, o usuário é desafiado a responder um conjunto de diferentes tipos de questões envolvendo resolução de problemas. O aplicativo foi construído para usuários do sistema operacional Android, que por ser um sistema operacional da Open Source (código livre), possui suas próprias tecnologias já integradas, que consequentemente, 
VI Congresso Brasileiro de Informática na Educação (CBIE 2017)

Anais do XXVIII Simpósio Brasileiro de Informática na Educação (SBIE 2017)

foram as utilizadas para implementar o T-mind. $\mathrm{O}$ aplicativo foi construído com interface responsiva baseada no material design da Google e pode ser executado em dispositivos (tablets ou smartphones) com versões a partir da 4.0. A interface de algumas telas do Tmind está disponível para consulta no seguinte link: interface do T-mind.

O T-mind foi construído tendo como público alvo alunos do ensino médio. Essa decisão foi tomada baseada nas diretrizes dos Parâmetros Curriculares Nacionais, no qual registram-se como uma das competências esperadas, além de outras, que o aluno a partir do $1^{\circ}$ ano do ensino médio seja capaz de "identificar regularidades em situações semelhantes para estabelecer regras, algoritmos e propriedades" [PCN 2002].

Para tornar o aplicativo mais atrativo, foram inseridos elementos gamificados os quais propõem o engajamento dos usuários na resolução dos exercícios. As estratégias de gamifiacação as inseridas no T-mind foram: (i) níveis de fases: básico, intermediário e avançado, onde cada nível possui 3 tipos de questões distintas: montagem (como mostrado na Figura 2 à esquerda), objetiva (como mostrado na Figura 2 ao centro) e resposta curta (Figura 2 à direita); (ii) sistemas de pontuação: premiação proporcional ao número de acertos à cada nível de complexidade; (iii) ranking: listagem dos usuários com pontuações mais elevadas; (iv) desafios: questões que incentivam o raciocínio lógico; (v) manual de regras: explicação detalhada sobre o uso do aplicativo.
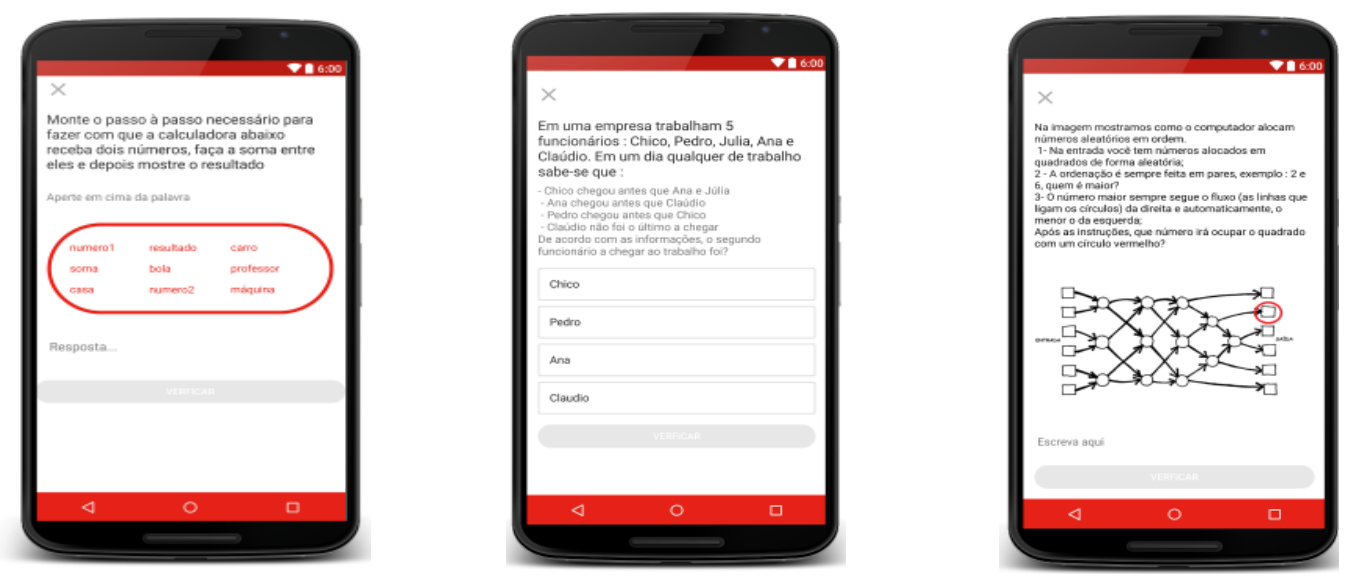

Figura 2. Tipos de questões do aplicativo: à esquerda, questões de montagem; ao centro, questões objetivas; à direita, questões de resposta curta.

\section{Resultados e Discussões}

Os resultados serão apresentados e discutidos sob duas vertentes: a análise do banco de questões (Seção 6.1) e a avaliação de usabilidade do T-mind (Seção 6.2).

\subsection{Análise das questões do T-mind realizada por especialistas}

A análise das questões realizadas pelos juízes teve o intuito de apontar quais habilidades associadas ao PC estão inseridas no contexto do aplicativo. O resultado apontou que há a presença de cinco habilidades (dentre as 9 avaliadas) relacionadas ao PC. Conforme mostra a Figura 4, no total de 20 questões, a habilidade de análise de dados foi a mais identificada pelos avaliadores (95\% das questões), seguidas pelas habilidades de coleta 
de dados ( $85 \%$ das questões), decomposição (75\% das questões), abstração (64\% das questões) e representação de dados (1\% das questões). Ressaltamos que a habilidade de construção de algoritmo também foi identificada pelos avaliadores, mas não a validamos, pois, a mesma não recebeu 3 votos na mesma questão.

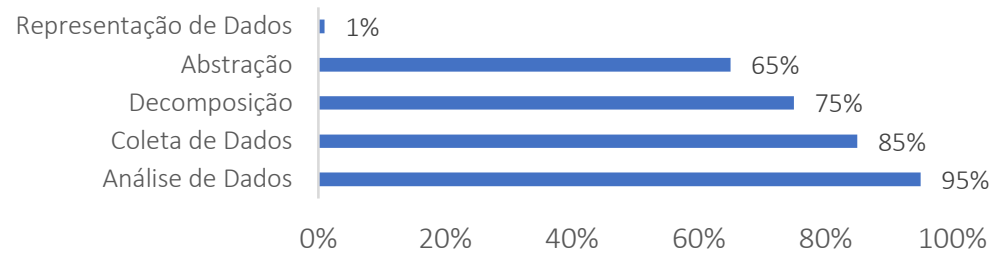

Figura 3. Avaliação dos especialistas sobre as habilidades presentes nas questões

Observando o resultado, percebemos que as habilidades de análise de dados, coleta de dados, decomposição e abstração foram identificadas em mais da metade das questões. Esse resultado pode estar associado ao fato dos graduandos que elaboraram as questões sentirem mais facilidade em criar questões explorando essas habilidades em detrimento as outras. Por outro lado, paralelização, generalização, simulação e automação, foram habilidades não encontradas pela maioria dos juízes. Isso pode ter ocorrido por dois motivos: $(i)$ os graduandos que elaboram as questões de forma colaborativa não conseguiram associar as habilidades ao contexto das questões, uma vez que são habilidades com características mais técnicas, associadas a conteúdo mais específico da Computação, como por exemplo, a programação; (ii) não houve uma compreensão adequada dessas habilidades por meio dos graduandos ao ponto de conseguir expressá-las em questões segundo os requisitos para o aplicativo.

\subsection{Análise da avaliação de usabilidade do T-mind}

Na segunda parte de avaliação do T-mind, analisamos as respostas dos 25 questionários respondidos pelos alunos. Quando perguntamos sobre a motivação em usar o aplicativo, fatores relacionados à pontuação, ao ranking e os desafios tiveram influência positiva em manter os alunos engajados no uso do aplicativo. A Figura 4 à esquerda mostra que $57 \%$ dos alunos avaliaram os elementos gamificados do T-mind como muito estimulante, $31 \%$ como estimulante, $8 \%$ como irrelevante e apenas $4 \%$ como pouco estimulante. Esse resultado já era esperado, pois durante a aplicação do questionário, foi observado que vários alunos se mostraram motivados em explorar o aplicativo bem como resolver as questões. Já quando fomos mais específicos e perguntamos se as questões do T-mind são desafiadoras, $46 \%$ dos alunos afirmam que são bastante desafiadoras, seguido por $37 \%$ afirmaram "sim", e 12\% afirmaram "mais ou menos".

Com relação à satisfação, no qual avaliou-se a interface, a Figura 5 à esquerda apresenta os resultados para a pergunta "o que você achou da interface do T-mind?" 59\% dos alunos avaliaram a interface do T-mind como ótima, 35\% como boa, 3\% como regular e apenas $1 \%$ dos alunos avaliou como ruim. Acredita-se que esse resultado seja devido a interface ter sido desenvolvida com conformidade com o material design da Google e as telas inspiradas no Duolingo. No entanto, o quesito eficiência, no qual avaliou-se o funcionamento do T-mind, teve resultado negativo. 30\% dos alunos avaliaram o funcionamento como regular, $20 \%$ e $9 \%$ como ruim e péssimo, respectivamente, como é 
VI Congresso Brasileiro de Informática na Educação (CBIE 2017)

Anais do XXVIII Simpósio Brasileiro de Informática na Educação (SBIE 2017)

ilustrado na Figura 5 à direita. O motivo dessa avaliação se deve ao fato que em dispositivos com menor recursos de hardware, o T-mind apresentou funcionamento mais lento, frustrando alguns alunos.
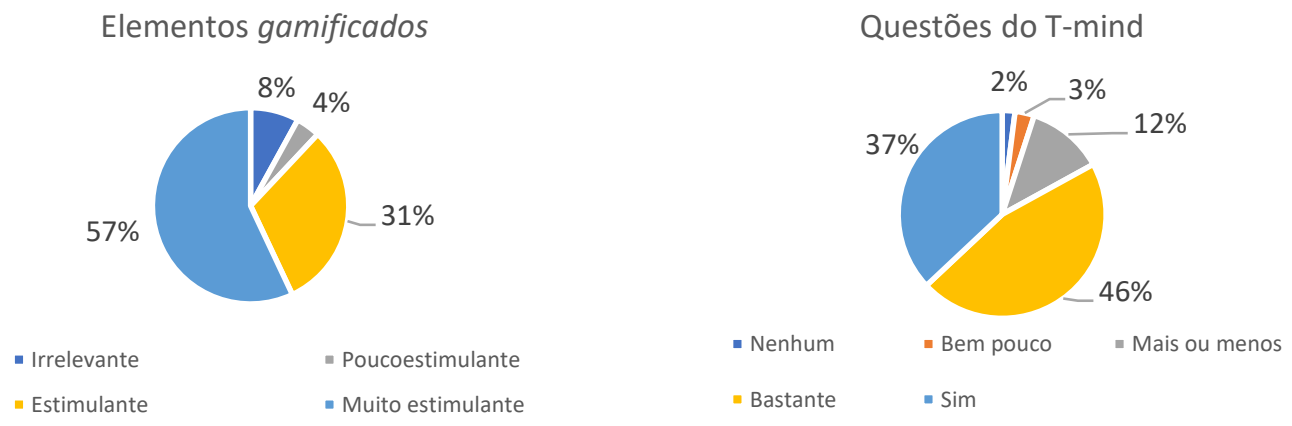

Figura 4. Resultados referentes a gamificação do T-mind
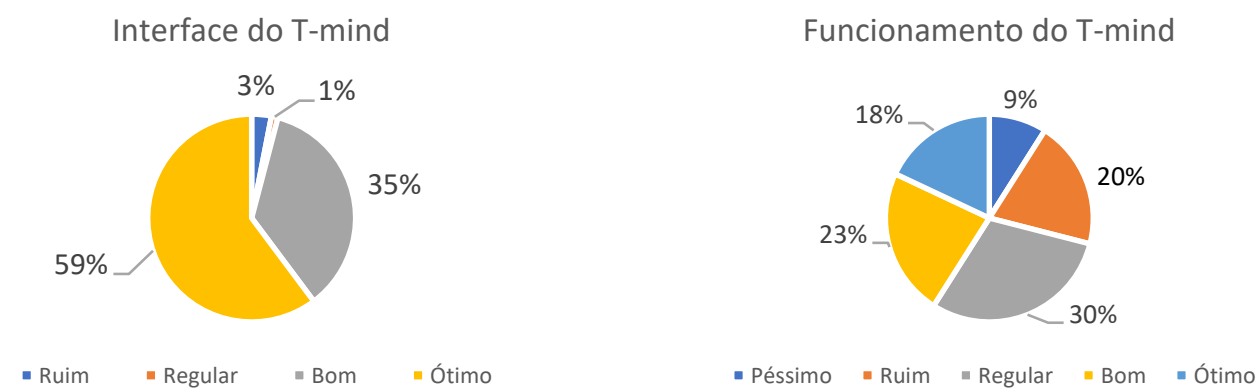

Figura 5. Resultados referentes a interface e funcionamento do T-mind

\subsection{Ameaças à validade}

Nossa pesquisa apresenta algumas ameaças à validade: $(i)$ as questões elaboradas de forma colaborativa podem não capturar seus respectivos efeitos; (ii) a amostra pequena de questões (iii) o baixo número de avaliadores das questões; (iv) o perfil da turma de alunos gostar de jogos; $(v)$ a avaliação da ferramenta realizada com apenas um método.

\section{Considerações finais}

Esse trabalho apresentou uma abordagem para estimular habilidades relacionadas ao PC por meio de um aplicativo gamificado, o qual não requer conhecimentos prévios de programação. Os resultados apontaram que as questões atuais do T-mind podem estimular cinco habilidades: análise de dados, coleta de dados, decomposição, abstração e representação de dados. Já o resultado da avaliação de usabilidade apontou que os elementos gamficados mantiveram o engajamento no uso do aplicativo. Entretanto, em dispositivos com recursos limitados de hardware, o T-mind apresentou um funcionamento lento, frustrando alguns alunos.

O uso de dispositivos móveis é uma tendência da sociedade contemporânea e o Tmind insere-se como uma proposta de aplicativo para estimular habilidades que possam auxiliar as pessoas na resolução de problemas no cotidiano. Como trabalhos futuros pretendemos aumentar o número de questões presentes no aplicativo, como também 
VI Congresso Brasileiro de Informática na Educação (CBIE 2017)

Anais do XXVIII Simpósio Brasileiro de Informática na Educação (SBIE 2017)

realizar um estudo detalhado sobre os efeitos das questões sobre os alunos. Outro estudo a ser explorado é a aplicação da ferramenta com outras faixas etárias.

\section{Referências}

Araujo, A. L., Andrade, W., \& Serey, D. (2016). Um Mapeamento Sistemático sobre a Avaliação do Pensamento Computacional no Brasil.. In Anais dos Workshops do CBIE (Vol. 5, No. 1, p. 1447).

Barr, V. \& Stephenson, C. (2011). Bringing computational thinking to k-12: What is involved and what is the role of the computer science education community? ACM Inroads, 2(1):48-54.

CSTA (2011). Operational Definition of Computational Thinking for K-12 Education. Disponível em: https:/c.ymcdn.com/sites/www.csteachers.org/resource/resmgr/ CompThinkingFlyer.pdf?hhSearchTerms $=\% 22$ computational + and + thinking $\% 22$. Acesso em: 10 jul 2017.

Falcão, T. P., \& Barbosa, R. (2015). "Aperta o Play!" Análise da Interação Exploratória em um Jogo Baseado em Pensamento Computacional. In Anais do SBIE. p. 419.

França, RS de, WC da Silva, and HJC do Amaral. "Ensino de ciência da computação na educação básica: Experiências, desafios e possibilidades." XX Workshop sobre Educação em Computação 2012.

Gomes, T., \& Alencar, A. (2015). Análise Empírica de Jogos Educativos para Dispositivos Móveis voltados a Disseminação do Pensamento Computacional na Educação Básica. In Anais dos Workshops do IV CBIE. p. 731.

IDC. (2015). Smartphone OS Market Share, 2015 Q2. Disponível em: http://www.idc.com/prodserv/smartphone-os-market-share.jsp. Acessado em 05 de Julho de 2017.

Kapp, K. M. (2012). The gamification of learning and instruction: game-based methods and strategies for training and education. John Wiley \& Sons.

Leffa, V. J. (2014). Gamificação adaptativa para o ensino de línguas. In Anais do Congresso IberoAmericano de Ciência, Tecnologia, Inovação e Educação. Buenos Aires pp. 1-12.

BRASIL. MINISTÉRIO DA EDUCAÇÃO. PCN (2002) Ensino Médio: Orientações Curriculares Complementares aos Parâmetros Curriculares Nacionais. Brasília: MEC/SEB, 2002.

Pinho, G., Weisshahn, Y., de Brum, C. F., Cavalheiro, G. G. H., \& Cavalheiro, S. (2016). Proposta de Jogo Digital para Dispositivos Móveis: Desenvolvendo Habilidades do Pensamento Computacional. In Anais do SBIE. Vol. 27, No. 1, p. 100.

Silva, Williane Rodrigues de Almeida, Aguiar, Yuska Paola Costa, Araújo, Juliana Aragão. (2016). Avaliação Tridimensional do uso do Scilab No Ensino De Matrizes. In Anais do XXI Congresso Internacional de Informática Educativa. p. 495.

Wing, J. M. (2006). Computational thinking. Communications of the ACM, 49(3), 33-35. 